\title{
Politique
}

Politique

\section{Léon Dion, Québec 1945-2000 Tome 1 -À la recherche du Québec, Québec, Les Presses de l’Université Laval, 1987, 182 p.}

\section{Guy Rocher}

Numéro 14, automne 1988

Sport et politique et Le NPD

URI : https://id.erudit.org/iderudit/040604ar

DOI : https://doi.org/10.7202/040604ar

Aller au sommaire du numéro

Éditeur(s)

Société québécoise de science politique

ISSN

0711-608X (imprimé)

1918-6584 (numérique)

Découvrir la revue

Citer ce compte rendu

Rocher, G. (1988). Compte rendu de [Léon Dion, Québec 1945-2000 Tome 1 la recherche du Québec, Québec, Les Presses de l'Université Laval, 1987, 182 p.] Politique, (14), 159-163. https://doi.org/10.7202/040604ar d'utilisation que vous pouvez consulter en ligne.

https://apropos.erudit.org/fr/usagers/politique-dutilisation/ 
Léon Dion, Québec 1945-2000 Tome $1-A$ À la recherche du Québec, Québec, Les Presses de l'Université Laval, 1987, 182 p.

Ce dernier ouvrage de Léon Dion est étonnant. Certains sans doute le trouveront déroutant, déconcertant; certains autres seront déçus, d'autres encore agacés ou irrités. Pourtant, c'est un Léon Dion qu'il faut connaître et fréquenter.

Le livre est étonnant à plusieurs égards: le ton, le discours, l'intention ne sont pas ce à quoi Léon Dion nous avait habitués dans ses publications antérieures. Le ton d'abord. Il est très personnel, intimiste même. Léon Dion y parle beaucoup et souvent de lui-même; il livre ouvertement, candidement même diraiton, ses opinions personnelles, ses sentiments les plus profonds et ceux aussi qu'il porte à fleur de peau, l'évolution de ses convictions politiques à travers l'histoire de sa vie depuis ses origines rurales, ses études à Québec et à l'étranger, sa "difficile insertion dans le Québec des années 1950» (page 9), jusqu'à ses réflexions d'aujourd'hui sur demain. Une telle franchise paraîtra à certains une forme d'exhibitionnisme agaçant. Mais passons!

Le discours aussi est inhabituel. Traitant du Québec, Dion cite bien sûr les sociologues qui en ont parlé et qui ont écrit sur le sujet: Jean-Charles Falardeau à qui il rend hommage - Fernand Dumont, Marcel Rioux, Marc-Adélard Tremblay (et Guy Rocher, excusez-moi!). Mais ceux que Dion cite le plus abondamment sont des littérateurs: romanciers, poètes, chansonniers. Ce sont en particulier Félix Leclerc, Gaston Miron (les deux auteurs préférés, si l'on se fie à l'Index), Michel Tremblay, Paul Chamberland, Hubert Aquin, Émile Nelligan. Fernand Ouellette, Gilles Vignault, Félix-Antoine Savard, Jacques Godbout, etc. Et il faut accorder 
une place particulière à André Laurendeau, dont la personnalité et la pensée ont joué un rôle important dans la vie de Léon Dion.

L'intention, enfin. Léon Dion n'est plus ici seulement l'analyste qui cherche à comprendre et à expliquer, suivant une méthode rigoureuse. Il s'affiche plutôt comme un militant, presque un activiste si l'on peut oser dire. Constatant le «sentiment de lassitude (qui) s'est emparé de bien des esprits", Dion veut apporter un message: "Je ne saurais taire que l'un de mes buts est de tenter de rallumer la flamme de l'espoir» (p. XI). Et de fait, il termine l'ouvrage sur une dernière envolée, où il livre - comme dans le fameux "rêve» d'avenir de Martin Luther King — sa vision du Québec de demain: «Debout, nous négocierons les clauses constitutionnelles garantissant à deux sociétés différentes, à deux peuples distincts, qu'ils coexisteront égaux, heureux, fiers et prospères - et pour longtemps» (p. 165). Dion va même jusqu'à dire aveu étonnant et trop peu habituel de la part d'un homme de science - qu'il ne faut pas chercher dans cet ouvrage une pensée trop rigoureuse, suivant une méthode stricte. "Nombre de mes propos relèvent du sentiment, non de la logique» ( $\mathrm{p}$. IX), nous avertit-il dès le départ, ajoutant qu'il ne dispose ni "d'un fil conducteur unique» ni de "théories explicatives ni d'hypothèses de départ» (p. IX et $\mathrm{X}$ et 131).

Il faut dire que le sujet de l'ouvrage se prête à l'exercice. Dion a voulu explorer "l'identité québécoise" dans ses différentes composantes. C'est ce qui l'a conduit du côté des poètes plus que de celui des politologues (à vrai dire, je crois qu'il n'en cite pas un, à l'exception peut-être de son ami John Meisel) et à faire de l'introspection plutôt que de l'induction. Il a, dit-il, "laissé la bride au cou de notre imaginaire» (p. 131), voulant dire par là que c'est à travers la prose, la poésie et la chanson des littérateurs qu'il est allé à la recherche de la perception de soi des Québécois.

Pourtant, l'homme de science en Léon Dion ne pouvait pas être mis complètement à l'écart de l'exercice. Au fond, sa pré- 
occupation méthodologique n'est pas complètement absente: elle ressort à la fin de l'ouvrage, dans un court chapitre d'«éléments de méthode», où Dion retrouve l'univers plus familier de l'analyste. Il ne peut s'empêcher de revenir à la méthode du «systémisme cybernétique» qu'il avait développée dans Dynamique de la société libérale (1972) et dont il dit modestement qu'il ne la présente pas nécessairement comme «la meilleure méthode possible», mais qu'elle l'a bien servi dans son enseignement et dans ses recherches empiriques (p. 135). Cette méthode, dit-il porte «la marque de Georges Gurvitch ». Pour ma part, je suggère qu'elle se rapproche bien plus du cadre conceptuel du système social de Talcott Parsons que de celui de Gurvitch. S'il est un théoricien qui a développé une théorie du système social et un cadre conceptuel systémique, et qui a été sensible à la cybernétique - sans trop lui emprunter - c'est bien plus Talcott Parsons que Georges Gurvitch. D'ailleurs, la notion du système social, de ses composantes, le type d'analyse que Léon Dion en propose (p. 135-140) rappellent vivement les grandes lignes de la sociologie de Parsons.

En tout cas, comme entrée en matière d'une trilogie qui s'annonce maintenant comme une tétralogie, cet ouvrage nous livre, comme Dion lui-même dit espérer qu'on le voie, «le fruit neuf d'un homme neuf». Léon Dion nous annonce trois autres Tomes à venir, qui constitueront l'ensemble de l'ouvrage intitulé Québec, 1945-2000. J'imagine que le ton et le discours des suivants ne seront plus tout à fait ceux de ce premier tome. Car en réalité, celui-ci est un accident; il est l'enfant non prévu. Pour entreprendre sa trilogie, Léon Dion s'est demandé ce qu'est le Québec et ce qu'est la personnalité québécoise. Les «quelques phrases bien senties» qui devaient répondre à cette question - la «question insoluble» - ont pris les proportions d'un livre complet. C'est probablement ce qui fait que le fruit inattendu a les richesses et les faiblesses d'un enfant de l'amour. Il présente cependant, me semble-t-il, un mérite certain, celui de voir un éminent politologue 
jeter un pont entre sa discipline et la littérature. Comme les litrérateurs québécois ont beaucoup et bien écrit sur le Québec, le politologue québécois est bien placé pour puiser à la source de l'imaginaire et en faire son profit. Je sais gré à Léon Dion d'avoir eu à la fois la modestie et l'intelligence de le reconnaître et d'avoir pris le risque que pouvait représenter pour lui l'aventure d'aller voguer sur des eaux étrangères. "Heureux qui comme Ulysse... ».

Mais cette démarche soulève un problème qui n'a pas été posé par Léon Dion et sur lequel on aurait aimé le voir réfléchir. C'est celui de la méthodologie que suppose l'intégration que fait le politologue du discours des littérateurs dans celui d'une discipline à vocation scientifique. Posé en termes simples, le problème s'exprime ainsi: «Y a-t-il lieu de faire une traduction du discours littéraire, lorsqu'on le transferre dans le langage des sciences sociales?» Si le politologue transpose directement le discours des romanciers et des poètes, il ne fait pas autre chose que ceux-ci, c'est-à-dire qu'il emploie le langage que Dion appelle de l'imaginaire, lequel recourt à une forme symbolique différente du langage des sciences sociales. Si le politologue opte plutôt pour traduire le symbolisme de l'imaginaire dans celui des sciences sociales, comment doit-il procéder à cette traduction? Quels sont les canons, les règles, les normes à suivre?

Léon Dion, me semble-t-il, va d'une option à l'autre, sans nous le dire et surtout sans s'interroger sur le problème sousjacent à sa démarche. Il paraît prendre pour acquis que les sciences sociales peuvent emprunter le symbolisme littéraire sans autre forme de procès. Pourtant, lorsqu'il analyse le discours des hommes et femmes politiques, ou celui des militants et militantes d'une cause, le politologue doit les décoder et les interpréter minutieusement. Le discours des romanciers et des poètes est lui aussi "engagé», militant, à cet égard contextué - a fortiori lorsqu'il évoque la crise de l'identité nationale. En ayant évité de s'interroger systématiquement sur ce problème, il me semble que Léon Dion 
a escamoté une difficulté méthodologique qui aurait mérité qu'il s'y arrêtât.

Guy Rocher

Université de Montréal 